\title{
Interactive comment on "Bromine Speciation in Volcanic Plumes: New in-situ Derivatization LC-MS Method for the Determination of Gaseous Hydrogen Bromide by Gas Diffusion Denuder Sampling" by Alexandra Gutmann et al.
}

Alexandra Gutmann et al.

alexandragutmann@web.de

Received and published: 3 July 2021

We thank the reviewer for his time to review this manuscript and the many suggestions for improvement.

Reviewer: General

The paper by Gutmann et al describes the evaluation and field deployment of a new technique to measure $\mathrm{HBr}$ in volcanic plumes via denuder sampling and subsequent 
analysis by LC-MS. Given the current reliance on $\mathrm{BrO}$ measurements obtained via remote sensing to characterize halogen chemistry in these plumes, the development of methods to measure $\mathrm{HBr}$ represents an important contribution to the field.

\section{Method description}

While the manuscript would be improved greatly by a characterization of the sensitivity and dynamic range of this technique, the authors do demonstrate the technique is selective for $\mathrm{HBr}$ and that the fraction of $\mathrm{HBr} /($ Total Bromine) can be obtained as a function of distance from the emission source which is a helpful quantity even if substantial uncertainties remain as to the actual amount of $\mathrm{HBr}$ measured.

While I believe this paper is still a useful contribution to the community, I do want to make clear that I am not convinced the authors have demonstrated an ability to quantitatively measure atmospheric $\mathrm{HBr}$ at the mole fractions reported. Below I provide some suggestions for improvement of the manuscript.

Response: There is indeed a large amount of uncertainty in many of these measurements, and we would like to address and discuss them. From the comments on the review, we also recognized that parts of the discussion were missing or could be developed further, and we have expanded or reworded the text as needed. Please see the details for the changes in the responses to the following comments below.

\section{Reviewer: Line 91: Why does $250 \mathrm{~mL} / \mathrm{min}$ provide "ideal" sampling efficiency?}

Response: One influence on the choice was that an optimal flow rate of $250 \mathrm{ml} / \mathrm{min}$ was determined for a previously developed denuder system for reactive bromine species (Rüdiger et al., 2017). The two denuder systems are supposed to complement each other and, in the best case, sample in parallel with the same pump. Even though a higher flow rate would be advantageous because more sample volume can be enriched, it is a logistical advantage if both systems sample at the same flow rate.

It was described in the introduction that under the given conditions with a flow rate of 
$250 \mathrm{ml} / \mathrm{min}$, the probability of gaseous $\mathrm{HBr}$ molecules diffusing to the denuder walls and reacting with the coating is $99.999997 \%$. With the breakthrough studies in section 3.2.1, it has also been practically proven that a slower flow is not necessary.

Nevertheless, it is true that the wording 'ideal' is misleading here and has been changed.

Changes: A sampling flow rate of $250 \mathrm{~mL} / \mathrm{min}$ was used.

Interactive

comment

Reviewer: Line 150: While I see the actual temperature programs are given in the supplement, it would be good to give the temperature ranges and total run times for the $\mathrm{GC}$ separations in the main text as well.

Changes: The oven temperature programs (range from $90-300{ }^{\circ} \mathrm{C}$ with total runtimes of 9-30 $\mathrm{min}$ ) and the ions used for quantification are summarized in the supplementary material (Table S1).

Reviewer: Line 239: Why this range of values? The highest value you report in Table 2 is $1.90 \mathrm{ppb}$. Are these high values atmospherically relevant? Does this relationship hold at lower mole fractions such as those you report?

Response: This comment highlights a clear gap in the comprehensibility of the experiments. The presentation of the test gas concentration alone was misleading here. Test gas concentrations were higher than expected in the atmosphere but sampling duration was shorter, resulting in a sampled amount of $\mathrm{HBr}$ in the same range as the field samples. For a better comparability the amounts of $\mathrm{HBr}$ (in $\mathrm{nmol}$ ) were added to the laboratory studies and field sample results.

Still, this will only reflect the higher concentrated data from the set shown here from Masaya, that's true. For upcoming samples from volcanic plumes where we expect higher bromine emissions, such as from Etna, the values may fit better.

Changes: Modified Table 3 and 4 
Reviewer: Figure 2: If the goal is to compare the two techniques using the Raschig Tube measurements as an independent variable in a calibration, an orthogonal distance regression of the two sets of measurements would more clearly demonstrate the comparability of the two sets of measurements and give the reader an idea of the sensitivity of the denuder measurements. As I point out above, the calibration should be performed over an atmospherically relevant range of values.

Response: Yes, the comparison with the approved alkaline method was used to prove the accuracy and, above all, the comparability of both methods, since a ratio is to be formed from their results.

We acknowledge the reviewer's comment about the need to discuss the comparison between the methods more thoroughly. We followed the suggestion to perform an orthogonal distance regression for comparing the results of both methods.

Changes: New Figure 4 (Fig. 1). On average, the results related to denuder sampling yielded $99 \pm 11 \%$ of the $\mathrm{HBr}$ values determined by the Raschig Tube. An orthogonal distance regression was performed and is shown in Fig. 4. Based on the line equation obtained, small values, such as those observed here in the field samples, can yield higher results from denuder determinations than expected via the Raschig Tube. We have concluded, that the $\mathrm{HBr}$ values determined by denuders in field samples can be considered a fraction of the total bromine determined by the Raschig Tubes. To account for the comparison studied, the deviation found is included as an error of the denuder field samples in Table 4.

Reviewer: Section 3.5: Move details of the instrument field deployment to the methods. It would be helpful to add information about how the pump was powered during sampling both on the ground and during UAV sampling to give the reader an idea of the logistical requirements. Since UAV sampling is covered, it would also be helpful to provide the approximate weight of the instrument configured for UAV deployment. Was the instrument the only payload for the UAV or was it flown with other instruments? I 
understand references are provided for the details of UAV campaigns and the answers may exist there, but it would be good to provide the answers to these questions in this paper.

Response: We agree with the reviewer that this additional information makes the manuscript easier to follow. The description of the fieldwork has been moved to the new section '2.4 Field application at Masaya 2016'. A brief description of the drone setups used has also been included here. The GilAir Plus used in ground-based sampling is an air sampling pump, commonly used for person-carried sampling of hazards at work. The pump already contains its own battery and weighs approximately $600 \mathrm{~g}$. In the manuscript, we have given the reference to the size and the included battery.

Changes: 2.4 Field application at Masaya 2016 A first set of field samples was collected between 18.-21. of July 2016 at the Santiago Crater of the Masaya volcano (Nicaragua). A detailed description of the location can be found in Rüdiger et al. (2021). In summary, sets from different methods were collected simultaneously together at changing locations with various distances (200-2000 m, Fig. 3b) to Masaya's emission source at Santiago crater (Fig. 3b).

A total of eight ground-based and two UAV-based samples for the newly developed denuder method are presented here. In ground-based sampling sets, two denuders were sampled in series (Fig. 3a). Both denuders were extracted and results were summarized. Sampling was performed by a Gilian GilAir Plus handheld pump (battery included) with a flow rate of $250 \mathrm{ml} / \mathrm{min}$ for about 1-1.5 hours for each denuder. In addition to EP-coated denuders, samples with 1,3,5-trimethoxybenzene coated denuders for the determination of reactive bromine. as well as Raschig Tubes as alkaline traps for the determination of total bromine and total sulfur were collected simultaneously side by side. The results of these samples can also be found in Rüdiger et al. (2021).

First drone-based samples were collected with an UAV using a small four-rotor multicopter with foldable arms (Black Snapper, Globe Flight, Germany) called RAVEN (Rüdi- 
ger et al., 2018). For the UAV-based sampling, a remotely controlled sampler (called Black Box) was used and is also described in detail in Rüdiger et al. (2018). The Black Box enabled logging of the sampling duration and SO2 mixing ratios via the built-in SO2 electrochemical sensor (CiTiceL 3MST/F, City Technology, Portsmouth, United Kingdom). The Black Box has $20 \times 14 \times 13 \mathrm{~cm}$. With this setup (Black Box + denuder) of approx. $1 \mathrm{~kg}$ we achieved flight times of up to $15 \mathrm{~min}$. In drone-based sampling flights, individual denuders were used with sampling times between 5-10 minutes.

Reviewer: Line 262-275: The authors haven't actually demonstrated that they can accurately quantify atmospheric $\mathrm{HBr}$ at low mole fractions as their laboratory studies only go from 3 to $20 \mathrm{ppb}$. Even if one assumes these data were quantitative below $3 \mathrm{ppb}$, the inclusion of actual values below LOD in Table 2 is also not appropriate, it should just be noted that the measurements were below the detection limit.

Response: The revised tables now show that the laboratory studies are at least in the range of the samples found and cover the larger values. Values below the LOD have been marked as such.

Changes: Modified Tables 3 and 4.

Reviewer: Line 282: Rephrase "This is in very good agreement with the results obtained with the model's estimations". The point of these comparisons is an evaluation of the model performance, not affirmation of your observations. The model being consistent with your observations reflects favorably on the model, but says nothing about the accuracy of your measurements as you imply here. This sentence as written implies you have doubts about the measurements. I would also add the model prediction to Fig. 5 for ease of comparison by the reader.

Response: We agree with the reviewer's suggestions that the statements had to be rephrased. In addition, the model run mentioned was taken over into the figure

Changes: Modified Figure 5 (Fig. 2). 
In the work of Rüdiger et al. (2021) the results of total bromine, total reactive bromine and bromine monoxide from accompanying methods were used to run the atmospheric box model CAABA/MECCA, which was initialized by a high-temperature equilibrium model. The model run that best described the data in Rüdiger et al. (2021) was used here for comparison and is highlighted in light blue for the ratio of $\mathrm{HBr} /$ total $\mathrm{Br}$ in Fig. 5. This run was based on a Br/S ratio of $7.4 \times 104$. The $\mathrm{Br} / \mathrm{S}$ ratio for the measurements considered here on 18.-21.7. was on averaged $6.2 \pm 1.0 \times 104$. Even though the general trend between measured values and model predictions is consistent, on average the measured values appear to be slightly higher than those calculated by this model run. Following the observations in Rüdiger et al. (2021), a cause may be the influence of aerosol. Aerosol was not measured simultaneously, smaller particle number concentrations and diameters than assumed may lead to slower $\mathrm{HBr}$ loss than expected. Also, deviation from the assumed wind speed can lead to a horizontal shift of the measurements while the deviations between Denuder and Raschig method cause a vertical shift. Overall, the trend observed would have to be confirmed by further samples. These samples give us a first idea that we can confirm our general idea about the $\mathrm{HBr}$ consumption. Of course, a solid foundation will require many field samples and further consideration of the two methods used and their joint application at the expected concentrations.

Reviewer: Fig. 5: As discussed previously, I think adding the model results referenced in the text would be helpful. The point markers should also be changed. Right now they can be confused with error bars, which is problematic. The author's should also add error bars on the ratio since they are presented in the caption. The y-axis label is not accurate. I believe what is being presented is the ratio of $\mathrm{HBr} /$ reactive bromine, not the ratio of $\mathrm{HBr}$ / bromine atoms which is what the label says

Response: We liked the suggestion to add the model run to the figure. It seems that the choice to add caps at the end of the error bars was confusing. They have now been removed to make the figure clearer. The $y$-axis labels have also been adjusted. What 
can be seen here is the ratio of $\mathrm{HBr}$ to total bromine, which was labeled only as $\mathrm{Br}$, misleading to bromine radicals. We changed to 'total Br' for clarification.

Changes: Modified Figure 5.

Please also note the supplement to this comment:

https://amt.copernicus.org/preprints/amt-2020-357/amt-2020-357-AC1-

Interactive

supplement.pdf

Interactive comment on Atmos. Meas. Tech. Discuss., doi:10.5194/amt-2020-357, 2020. 
AMTD

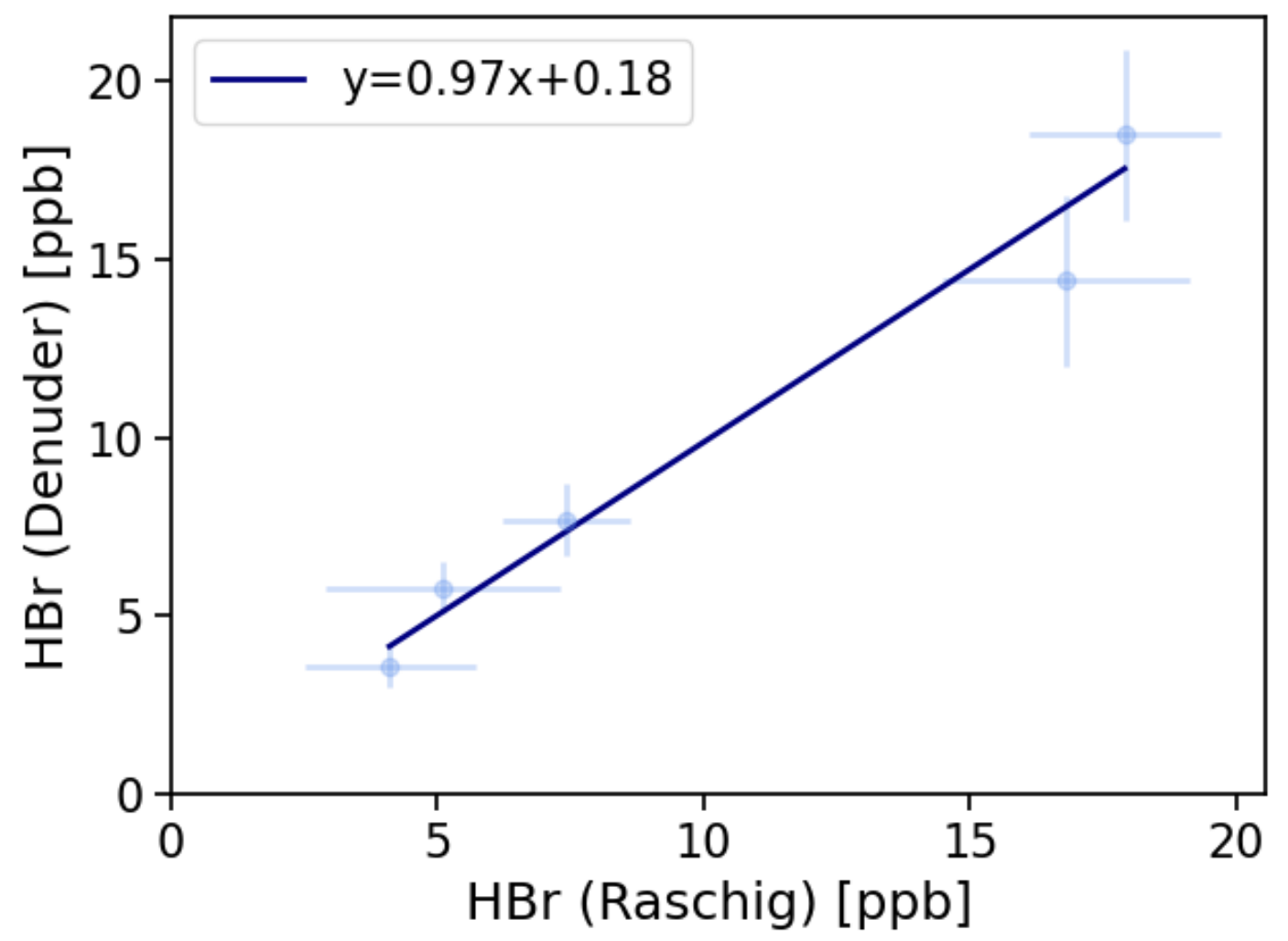

Interactive comment

Fig. 1. = Figure 4: Figure 4: Denuder results are plotted against Raschig results. The orthogonal distance regression model resulted in: $y=0.97( \pm 0.10){ }^{*} x+0.18( \pm 0.10)$ and a residual variance of 0.25 . 
AMTD

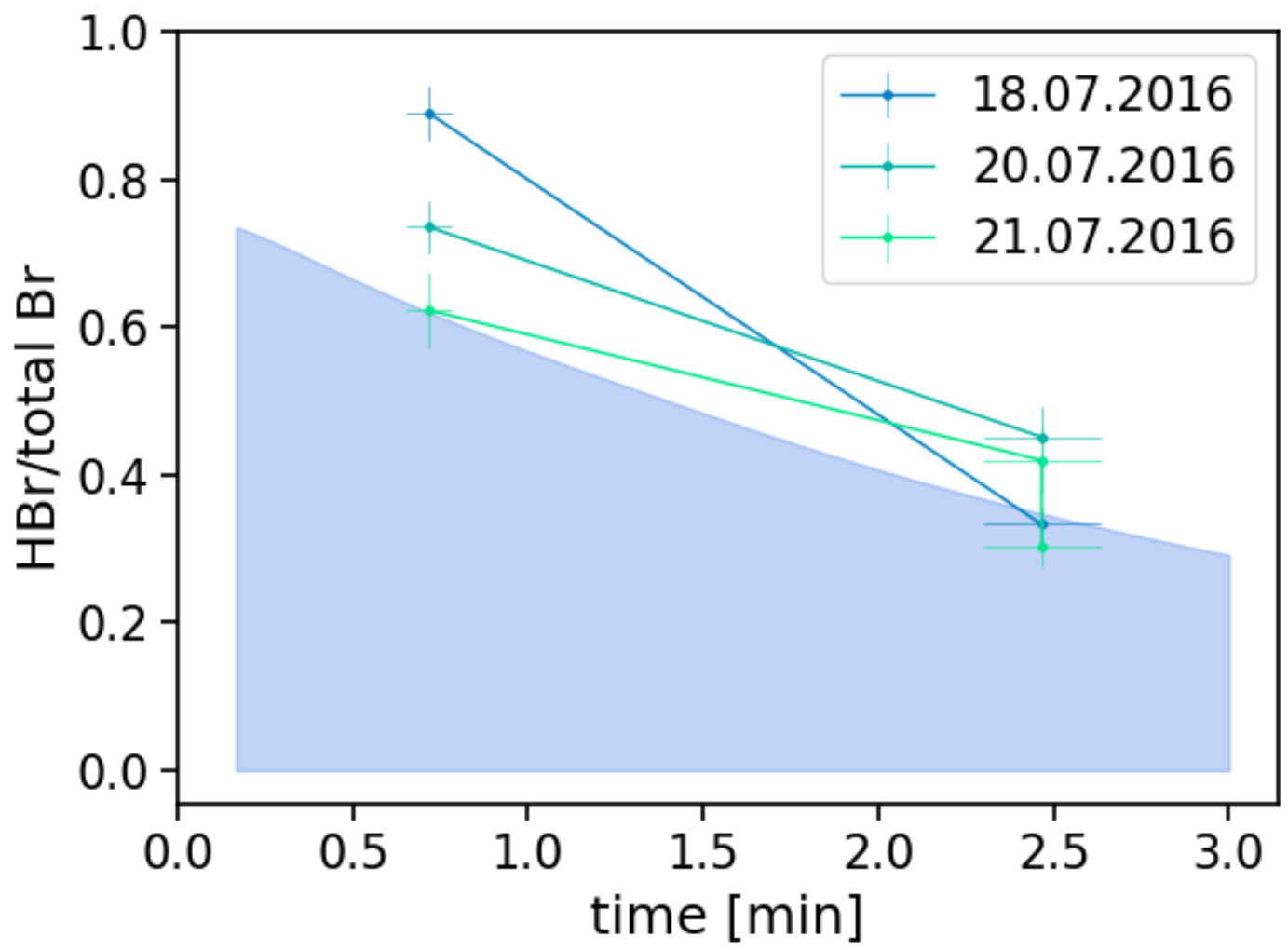

Interactive comment

Fig. 2. = Figure 5: Fraction of $\mathrm{HBr}$ (determined by EP-coated denuders) to total bromine (determined by Raschig Tubes). Assuming a windspeed of $5 \mathrm{~m} / \mathrm{s}, \mathrm{HBr}$ fractions decrease on average from $0.75 \pm 0.11$ at 0.7 